\title{
Coconut oil as a virucidal agent: prospects and challenges in COVID-19
}

\author{
S V Ramesh ${ }^{1}$ \\ ${ }^{1}$ Affiliation not available
}

May 7, 2020

\begin{abstract}
COVID-19 caused by the novel SARS Coronavirus-2 (SARS-CoV-2) is causing serious blockades in the global public health sphere. In the absence of specific antiviral treatment, exploration of plant-based products with antiviral potential has gained attention. We present the research prospects of utilizing coconut oil directly or its derivatives such as monolaurin in treating COVID-19 with a special emphasis on the biochemical characteristics features. The potential pitfalls therein and way forward are also highlighted. Possibility of developing a medium chain fatty acid-based nasal spray as a prophylactic or therapeutic is also discussed. Nevertheless, the potential impediments in devising suitable therapeutic models are also presented.
\end{abstract}

\section{Introduction}

Coconut (Cocos nucifera L.) is a tropical palm which is aptly called 'tree of life' (kalpavriksha) as it supplies wide range of food products of human consumption viz ., coconut oil, tender nut water, endosperm, inflorescence-sap derived sugar etc. Among this coconut oil has been attributed with many nutritional and health-conferring properties owing to its unique fatty acid composition (Dayrit 2014). The principal biochemical components of coconut oil and their potential health benefits are presented in Table 1 (Table 1 near here). Coconut oil is rich in medium chain triglycerides (MCTs) due to the predominance of C6 to C12 fatty acids attached to the glycerol moiety. Coconut oil derived-MCTs are easily soluble and digestible by salivary and pancreatic lipases in comparison to triglycerides of other vegetable oils which are predominantly long chain triglycerides (LCTs). Lauric acid (C12) is one of the prime medium chain fatty acids (MCFAs) and its content in coconut oil varies from 45-53\% (Dayrit 2014). Around $60 \%$ of the coconut triacyl glycerol (TAG) comprises MCFAs (both lauric acid and capric acid) in its positions $s n-1$, and $s n$ 3 (Dayrit 2014). Studies have demonstrated that proportion of dietary MCTs determines their mode of absorption as LCFAs predominantly enter the lymphatic system whereas MCTs are absorbed through portal veins (Mc Donald et al., 1980). Differential absorption of MCTs ensures their faster digestion, absorption and rapid energy expenditure thereby alleviating adiposity in humans (St-Onge et al., 2003). Besides their hypolipidemic effects, MCTs are known to have antimicrobial effects. Most conspicuously, lauric acid and its monoglyceride derivative, monolaurin, have been demonstrated to possess anti-microbial properties against gram positive bacteria (Projan et al 1994, Peterson et al., 2004), and many viruses such as Junin virus (JUNV), vesicular stomatitis virus, Simian immunodeficiency virus (SIV) etc. infecting humans (Thormar et al., 1987; Hornung et al., 1994; Bartolotta, et al., 2001; Duke 2009; Li et al., 2009). Administration of coconut oil has been found to be an effective remedy for the treatment of many of the enveloped viruses such as such as Cytomegalovirus, Epstein-Barr virus, hepatitis C virus, influenza virus, leukemia virus (Enig 2010; Dayrit 2000; Fife 2013). The antiviral property of monolaurin has been attributed to its common role in disrupting the phospholipid layers in the membranes of the enveloped viruses causing their ultimate disintegration. Monolaurin has adverse effects on the virus assembly and maturation phases of infection cycle eventhough the viral RNA and protein synthesis are unimpeded (Hornung et al., 1994). The binding of 
viral M protein (membrane) to the host cells was compromised due to the presence of lauric acid (Hornung et al., 1994). Supplementation of MCTs such as lauric acid induces cellular biosynthesis of triacylglycerols (TAG) in the infected cells (Hornung et al., 1994; Bartolotta, et al., 2001). This spurt in the biosynthesis of TAGs deprives the viruses of its crucial process, glycoprotein insertion in the plasma membrane, consequently interrupting the virus maturation process (Hornung et al., 1994; Bartolotta, et al., 2001). However, direct inactivation of virion particles of other enveloped viruses such as HIV and HSV due to physical contact with MCFAs is also not uncommon (Kristmundsdottir et al., 1999; Kohn et al., 1980). Antiviral properties of coconut oil-derived lauric acid or direct application of coconut oil have been established in animal studies. Administration of virgin coconut oil ( $\mathrm{VCO}$ ) through feed has enhanced the immunity parameters of chickens following challenge test with Avian influenza A virus subtype H5N1 (Yuniwarti et al., 2015).

COVID-19 (Coronavirus disease-2019), the pandemic caused by novel SARS Coronavirus (SARSCoV-2) has been wreaking havoc in the global health sphere. At this point of time no specific drugs are therapeutic agents are prescribed to control the disease eventhough clinical trials are ongoing. As the number of confirmed COVID-19 cases are increasing (3.517 million cases and death toll of $2,43,401$ as on 5/ May/2020) (https://www.who.int/docs/default-source/coronaviruse/situationreports $/ 20200505$ covid-19-sitrep-106.pdf?sfvrsn=47090f63_2) lot of interests have been generated towards identification of compounds of plant origin with antiviral potential. These developments have renewed the interests in studying coconut oil and its variant virgin coconut oil (VCO) against COVID-19. Philippine Council for Health Research and Development (PCHRD) and Department of Science and Technology (DOST) has announced a hospital-based project entitled "Virgin Coconut Oil and Omega-3a Adjunctive Therapy for Hospitalized Patients with COVID-19" (https://www.covid19.gov.ph/dost-to-study-benefits-ofvirgin-coconut-oil-on-covid-19-patients). In this context, this communication presents the pros and cons of developing the coconut oil and its products-based treatment for COVID-19 with special emphasis on biochemical basis of antiviral properties.

\section{Lauric acid vs. SARS-CoV-2}

In this milieu, it is imperative to assess the suitability of coconut oil-derived lauric acid in treating COVID 19 caused by SARS-CoV-2. The potential positives for this proposition are SARS-CoV-2 is an enveloped virus with characteristic lipid membrane having S-glycoproteins (spike proteins) over its surface. The membrane disrupting role of lauric acid or its derivatives in the presence of SARS-CoV-2 is yet to be investigated. Considering the significance of early viral infection phase, wherein the virus encoded S-proteins effectively binds the human receptor protein angiotensin converter enzyme 2(hACE2), any disruption of viral membrane lipids could be detrimental to the viral infection process and thus could prevent the entry of virus into human cells. In this context, development of coconut oil based nasal spray either alone or in combination with other plant-derived essential oils, wherein the spray composition with anti-microbial, anti-inflammatory and decongestant attributes shows the great potential (Carolyn et al. 2012; Willimann 2018). Fatty acid molecules, preferably lauric acid and capric acid (both of which accounts for $>60 \%$ of total fatty acids composition in coconut oil) have been developed into pharmaceutical formulations as mouth and throat rinse, nose and throat spray or nasal drops suitable for the treatment of respiratory syncytial virus (RSV). The efficacy of these virucidal formulations in decreasing the nasal viral load was demonstrated in mouse models without any off-target effects (Wyde et al., 2003). Thus, these instances highlight that development of lauric acid-based novel prophylactic or therapeutic measures would be of value to block the respiratory infections such as COVID-19 (Fig.1 ).

\section{[Insert Fig. 1 here ]}

Lauric acid as a food supplement was demonstrated to show no signs of toxicity on biochemical, histopathological profiles of animal models even at the dosage of $2,000 \mathrm{mg} / \mathrm{kg}$ body weight (Khan et al., 2020). Hence, considering the safety profile of lauric acid and coconut oil they can be recommended for satisfactory antiviral therapeutic purposes. It is further corroborated by the development of a proprietary antiviral formulation based on a short chain fatty acid (SCFA) (caprylic acid), ViraSAL. It's efficacy in treating the enveloped viruses such as Epstein-Barr, measles, herpes simplex, Zika and orf parapoxvirus, together with Ebola, Lassa, 
vesicular stomatitis and SARS-CoV-1 pseudoviruses were proven in vitro and in vivo studies (Fletcher et al., 2020). These investigations further underscore the importance of SCFAs and MCFAs in the treatment of enveloped viruses due to its surfactant activity on the virus envelopes. Although monolaurin is widely available and generally recognized as safe (GRAS) by US-FDA, the chemical synthesis of coconut lauric acid-based monosaccharide esters and their anti-microbial characteristics are encouraging. These monosaccharide lauric acid esters are biodegradable, non-toxic and have wide medical applications yet antiviral properties are not proven (Mutmainah et al., 2019). Sodium lauryl sulfate (SLS) - a tensoactive agent which has detergent properties, is made from lauric acid. The broad spectrum surfactant activity of SLS has been instrumental in inactivating the human and animal infecting viruses such as human immunodeficiency virus (HIV), human papillomavirus (HPV) and herpes simplex virus (HSV) (Piret et al., 2002) and in demonstrating microbiocidal activities against the viruses infecting small ruminants (de Souza 2019). A functional monoglyceride (MAG) has been developed by esterifying lauric acid and its anti-microbial activity was demonstrated while using it as a food preservative (Luna et al., 2020).

Furthermore, investigations have shown that monolaurin has anti-inflammatory effects in rhesus monkey models in response to HIV-1 and SIV infections (Li et al., 2007). Monolaurin suppresses inflammatory immune responses including the repression of IL-6 (Interleukin -6) production. Zhang et al (2017) has attributed this effect to the disrupted lipid dynamics in the T-lymphocytes. The effect of monolaurin in lowering the levels of IL- 6 in COVID-19 patients opens a new avenue for the designing of novel therapeutics to block SARS-CoV-2 since it has been demonstrated that production of pro-inflammatory cytokines such as IL-6 leads to extreme inflammation of bodily parts resulting in acute respiratory distress syndrome (ARDS) associated with the disease (Liu et al., 2019). Inhalation of virgin coconut oil (VCO) reduces the inflammatory responses and relieves asthma related symptoms in rabbit models of allergic asthma (Kamalaldin et al., 2017) further substantiating the therapeutic potential of coconut oil. Nevertheless, prophylactic application of VCO did not evince any relief for allergic asthma (Kamalaldin et al., 2017).

\section{VCO and its antiviral property}

In HIV-infected human subjects, dietary supplementation of virgin coconut oil (VCO) for 6 weeks has improved the CD4+ T lymphocyte counts implying immune-modulatory role of VCO consequently disrupting HIV disease progression (Widhiarta 2016). Though WHO is wary of antiviral therapeutic potential of VCO in the absence of scientific evidence, Philippine's DOST is conducting two clinical trials on COVID-19 positive patients and COVID-19 persons under investigations (PUIs) by incorporating VCO as a dietary supplement in addition to the current drug regimen (https://www.pna.gov.ph/articles/1098482). As a consequence, antiviral properties and reduction of inflammatory responses due to lauric acid requires to be further investigated in human trials to further the use of coconut oil-derivatives in the therapeutics.

\section{Challenges in the current standard drug regimen}

The role of cellular $\mathrm{pH}$ is an important factor to consider while using lauric acid as a therapeutic agent. Research has revealed that acidic condition ( $\mathrm{pH} 4.2$ ) was found to be more effective than $\mathrm{pH} 7.0$ when MCFAs and long chain fatty acids (LCFAs) were evaluated for antiviral activities against RSV and parainfluenza virus type 2, as well as on HSV types 1 and 2 (Hilmarsson et al., 2007). Nonetheless, efficacy of the chemical drug chloroquine and its derivatives, currently being widely used for the treatment of COVID-19, functions by increasing the endosomal $\mathrm{pH}$ and disrupts the glycosylation of cellular receptor of the virus (Cortegiani et al., 2020). Hence, the compatibility and efficacy of coconut oil-and /or its derivative-based treatment in view of the current drug regimen are researchable concerns. Multi-targeted interventions to treat COVID-19 are gaining momentum so as to harvest the potential synergies and additive effects of various drug molecules (Gaborit et al., 2020). Hence, the optimal spatio-temporal administration of drug components including coconut oil and its products requires to be explored so that a combination therapy is designed to treat the COVID-19.

Recent evidences highlight the fact that cardiovascular co-morbidities associated with the COVID-19 is due to the SARS-CoV-2 infection of human endothelial cells and the resultant endotheliitis (Varga et al., 2020). 
This study thus corroborates the rationale for stabilizing the endothelial tissues while administering antiviral drugs for COVID-19 more so for the vulnerable patients with endothelial dysfunction. In this framework, it is relevant to acknowledge that lauric acid or coconut oil has an inherent positive impact by ameliorating the cardiovascular diseases and in fighting obesity (Assunção et al., 2009, Adeyemi et al., 2020). Hence, the need for supplementary designer therapies to stabilize the endothelial tissues would become redundant when lauric acid or coconut oil is used in COVID-19 treatment.

\section{Concluding remarks}

To conclude, there are research evidences to prove the antiviral effects of coconut oil and monolaurin in animal and human subjects. However, large scale human trials to treat COVID-19 followed by rigorous analysis are mandatory to ascertain to the therapeutic potential of the coconut oil and its products. Providing due credits to the virus containment and other socio-economic measures of the local government, the successful turnaround in the containment of COVID19 in the Indian state of Kerala (as on 19/April/2020) is tempting us to correlate it with the consumption of lauric acid by the population. Coconut oil is a major dietary component in this part of the world. It would be premature to conclude thus until a thorough epidemiological research investigating the effects of coconut oil-derived lauric acid and reduction of viral load in human subjects of Kerala proves it so. Furthermore, available evidences and proven food safety of coconut oil and its derivatives convinces us to safely conclude that the products of Cocos nucifera L. could form a supplemental therapeutics candidate, if not principal antiviral agent, for COVID-19.

\section{Conflict of Interest Statement:}

Authors would like to declare that no competing interests exist

\section{Funding :}

None

\section{References :}

1. Adeyemi, W.J., Olayaki, L.A., Abdussalam, T.A., Toriola, A.P., Olowu, A.B., Yakub, A.J. and Raji, A.O. (2020). Investigation of the effects of dietary modification in experimental obesity: low dose of virgin coconut oil has a potent therapeutic value. Biomedicine \& Pharmacotherapy, 126 :110110.

2. Arlee, R., Suanphairoch, S., Pakdeechanuan, P. (2013). Differences in chemical components and antioxidant-related substances in virgin coconut oil from coconut hybrids and their parents. Food Res Int.20(5) : 2103-2109.

3. Assunção, M.L., Ferreira, H.S., dos Santos, A.F., Cabral, C.R., Florêncio, T.M.M.T. (2009). Effects of dietary coconut oil on the biochemical and anthropometric profiles of women presenting abdominal obesity. Lipids, 44 :593-601

4. Bartolotta, S., Garc'1a C. C., Candurra N. A., and Damonte E. B. (2001). Effect of fatty acids on arenavirus replication: inhibition of virus production by lauric acid. Arch Virol, 146 : 777-790

5. Carolyn et al. (2012). US Patent-https://patents.google.com/patent/US20120219644A1/en

6. Dayrit, C.S. (2000). Coconut oil in health and disease: its and monolaurin's potential as cure for HIV/AIDS. Ind Coconut J (Cochin),31:19-24.

7. Dayrit, F. (2014). The properties of lauric acid and their significance in coconut oil. J Am Oil Chem Soc. 92 :1-15 Doi. 10.1007/s11746-014-2562-7

8. de Sousa, A.L.M., Pinheiro, R.R., Araújo, J.F., de Azevedo, D.A.A., Peixoto, R.M., Andrioli, A., da Cruz Silva Bezerra, S.T. and da Silva Teixeira, M.F. (2019). Sodium dodecyl sulfate as a viral inactivator and future perspectives in the control of small ruminant lentiviruses. Arquivos do Instituto Biológico, 86 . doi. 10.1590/1808-1657000752018. doi: https://doi.org/10.1101/2020.03.29.009464

9. Duke, W. (2009). Medicine: Cocos nucifera Folk Medicine.

10. Enig, M. G. (2010). Health and nutritional benefits from coconut oil and its advantages over competing oils. Indian Coconut Journal. 9-15.

11. Enig, M.G. (1998). Lauric oils as antimicrobial agents: theory of effect, scientific rationale, and dietary 
applications as adjunct nutritional support for HIV-infected individuals. In: Nutrients and Foods in AIDS (Watson RR, ed.). CRC Press, Boca Raton, FL, USA, 81-97

12. Fife, B. (2013). Health properties of coconut oil. Agro Food Ind Hi Tec, 24(3 ): 7-10

13. Fletcher, N.F., Meredith L.W., , Tidswell E., Bryden S.R., Gonçalves-Carneiro D., Chaudhry Y., Lowe C.S., Folan M.A, Lefteri D.A., Pingen, M., Bailey D., McKimmie C.S., Baird A.W. (2020). A novel antiviral formulation inhibits a range of enveloped viruses. bioRxiv 2020.03.29.009464

14. Gaborit B.J., Bergmann J-F., Mussini C., Arribas J.R., Behrens G., Walmsley S., Pozniak A., Raffi F., (2020). Plea for multitargeted interventions for severe COVID-19. Lancet Infect Dis . https://doi.org/10.1016/S1473-3099(20)30312-1

15. Hilmarsson, H. (2008). Microbicidal activity of lipids, their effect on mucosal infections in animals and their potential as disinfecting agents, PhD Thesis, Faculty of Life and Environmental Sciences, University of Iceland, Reykjavik

16. Hilmarsson, H., Traustason, B.S., Kristmundsdottir, T. and Thormar, H. (2007) .Virucidal activities of medium- and long-chain fatty alcohols and lipids against respiratory syncytial virus and parainfluenza virus type 2: comparison at different $\mathrm{pH}$ levels. Arch. Virol.,152 :2225-2236

17. Hornung, B., Amtmann E., \& Sauer, G. (1994). Lauric acid inhibits the maturation of vesicular stomatitis virus. Journal of General Virology,75 (2): 353-361.

18. Kamalaldin, N. A., Sulaiman, S. A., Yusop, M. R., \& Yahaya, B. (2017). Does inhalation of virgin coconut oil accelerate reversal of airway remodelling in an allergic model of asthma?. International Journal Of Inflammation, Article ID 8741851. https://doi.org/10.1155/2017/8741851

19. Khan, H.U., Aamir. K., Sisinthy, S.P., Nagojappa, N.B.S., Arya, A. (2020). Food additive "lauric acid" possess non-toxic profile on biochemical, haematological and histopathological studies in female Sprague Dawley (SD) rats. PeerJ 8 :e8805 https://doi.org/10.7717/peerj.8805

20. Kohn, A., Gitelman, J., Inbar, M. (1980). Unsaturated free fatty acids inactivate animal enveloped viruses. Arch Virol, 66 : 301-307

21. Kristmundsdottir, T., Arnadottir, S.G., Bergsson, G., Thormar, H. (1999). Development and evaluation of microbicidal hydrogels containing monoglyceride as the active ingredient. J Pharm Sci88 : 1011-1015

22. Li, Q., Estes, J. D., Schlievert, P. M., Duan, L., Brosnahan, A. J., Southern, P. J., .. \& Nephew, K. R. (2009). Glycerol monolaurate prevents mucosal SIV transmission. Nature, 458(7241 ): 1034-1038. doi:10.1038/nature07831

23. Liu, T., Zhang, J., Yang,Y., Ma ,H., Li, Z., Zhang, J., Cheng, J., Zhang, X., Zhao, Y., Xia, Z., Zhang, L., Wu,G., Yi, J. (2020). The potential role of IL-6 in monitoring severe cases of Coronavirus disease 2019. medRxiv doi: 10.1101/2020.03.01.20029769

24. Luna, P., Hoerudin, Habiddin, Andarwulan, N. (2020). Characterisation of functional monoglyceride and its potential application. AIP Conference Proceedings 2215, 070006 https://doi.org/10.1063/5.0000576

25. McDonald, G.B., Saunders, D.R., Weidman, M., Fisher, L. (1980) Portal venous transport of long-chain fatty acids absorbed from rat intestine. Am J Physiol 239 :G141-G150

26. Mikołajczak N. (2017). Coconut oil in human diet - nutrition value and potential health benefits. Journal of Education, Health and Sport7(9): 307-319. eISSN 2391-8306. doi. http://dx.doi.org/10.5281/zenodo.997464

27. Mutmainah., Jumina., and Purwono, B. (2019). Chemical synthesis of monosaccharide lauric acid esters as antibacterial and antifungal agents. Materials Science Forum, 948 , 63-68. https://doi.org/10.4028/www.scientific.net/MSF.948.63

28. Peterson, M.L., Schlievert, P.M. (2006). Glycerol monolaurate inhibits the effects of Gram-positive select agents on eukaryotic cells. Biochemistry 45(7): 2387-2397

29. Piironen, V., Lampi, A.M. (2004). Occurrence and Levels of Phytosterols in Foods. (w:) Phytosterols as Functional Food Components and Nutraceuticals Dutta P.C. (Ed.). Marcel Dekker INC, Nowy Jork, $1-32$

30. Piret, J., Déseomeaux, A., Bergeron, M.G., et al., (2002). Sodium lauryl sulfate, a microbicide effective against enveloped and nonenveloped viruses. Current Drug Targets, 3(1): 17-30. 
31. Projan, S. J., Skrobot, S. B., Schlievert, P.M., Vandenesch F., and Novick, R. P. (1996). Glycerol monolaurate inhibits the production of beta-lactamase, toxic shock toxin1, and other staphylococcal exoproteins by interfering with signal transduction. Journal of Bacteriology, 176 : 4204-4209.

32. St-Onge, M-P., Ross, R., Parsons, W.D., Johns, P.J.H. (2003). Medium chain triglycerides increase energy expenditure and decrease adiposity in overweight men. Obes Res Clin Pract. 11(3): 395-402

33. Thormar, H., Isaacs, C.E., Brown, H.R., Barshatzky, M.R., Pessolano, T. (1987). Inactivation of enveloped viruses and killing of cells by fatty acids and monoglycerides. Antimicrobial Agents and Chemotherapy. 31(1): 27-31. doi:10.1128/AAC.31.1.27

34. Varga Z., , Flammer A.J., , Steiger P., Haberecker M., Andermatt R., Zinkernagel A.S., Mehra M.R, Schuepbach R.A., Ruschitzka F., Moch H. (2020). Endothelial cell infection and endotheliitis in COVID19. The Lancet . Published online April 17, 2020 https://doi.org/10.1016/S0140-6736(20)30937-5

35. Widhiarta, K. D. (2016). Virgin Coconut Oil for HIV - Positive People. Cord, 32 (1): 50-57.

36. Willimann (2018) US patent-https://patents.google.com/patent/US10307452B2/en

37. Wyde, P.R., Chetty, S.N., Timmerman, P. et al. (2003). Short duration aerosols of JNJ 2408068 (R170591) administered prophylactically or therapeutically protect cotton rats from experimental respiratory syncytial virus infection. Antiviral Res. $60: 221-231$

38. Yuniwarti E.Y. W., Asmara W., Artama W.T. and Tabbu C.R. (2015). Virgin coconut oil supplementation increased the survival of avianinfluenza virus (H5N1) infected chicken. Asian Journal of Poultry Science, 9 (2): 106-111

39. Zhang, M.S., Sandouk, A., Houtman, J.C. (2017). Glycerol monolaurate (GML) inhibits human T cell signaling, metabolism, and function by disrupting lipid dynamics. The Journal of Allergy and Clinical Immunology. 139(2 ): AB269-AB269. doi:10.1016/j.jaci.2016.12.866

Table 1. Biochemical components of coconut oil and their potential health benefits ( Adapted from Mikołajczak, 2017)

\begin{tabular}{|c|c|c|c|}
\hline Sl. No & Components & Percentage mean values & Potential health benefits \\
\hline \multirow[t]{11}{*}{1} & Fatty acid profile & & \\
\hline & C6:0 Caproic acid & 0.50 & Coconut oil predominantly comprise medium ch \\
\hline & C8:0 Caprylic acid & 6.76 & \\
\hline & C10:0 Capric acid & 6.37 & \\
\hline & C12:0 Lauric acid & 47.10 & \\
\hline & C14:0 Myristic acid & 17.19 & \\
\hline & C16:0 Palmitic acid & 8.80 & \\
\hline & C18:0 Stearic acid & 3.03 & \\
\hline & C18:1 Oleic acid & 6.45 & \\
\hline & C18:2 Linoleic acid & 1.45 & \\
\hline & C18:3 Linolenic acid & 0.27 & \\
\hline \multirow[t]{4}{*}{2} & Sterols & & \\
\hline & campesterol & 7.20 & Anti-cancer and hypocholesterolemic effects (Pii \\
\hline & stigmasterol & 12.30 & \\
\hline & $\beta$-sitosterol & 38.97 & \\
\hline \multirow[t]{9}{*}{3} & Tocols (tocopherols and tocotrienols) & & Natural anti-oxidants, components of cell memb \\
\hline & $\alpha$-Tocopherols & 1.01 & \\
\hline & $\beta$-Tocopherols & 0.13 & \\
\hline & $\gamma$-Tocopherols & 0.06 & \\
\hline & $\Delta$-Tocopherols & 0.20 & \\
\hline & $\alpha$-tocotrienols & 2.05 & \\
\hline & $\beta$ - tocotrienols & 0.09 & \\
\hline & $\gamma$ - tocotrienols & 0.49 & \\
\hline & $\Delta$ - tocotrienols & 0.05 & \\
\hline 4 & Phenolic acids ( $\mathrm{mg} / \mathrm{Kg}$ of oil) & & Natural anti-oxidants scavenging reactive oxyg \\
\hline
\end{tabular}




\begin{tabular}{|c|c|c|c|}
\hline Sl. No & Components & Percentage mean values & Potential health benefits \\
\hline & protocatechuic acid & 0.03 & \\
\hline & vanillic acid & 0.82 & \\
\hline & caffeic acid & 1.05 & \\
\hline & syringic acid & 1.87 & \\
\hline & ferulic acid & 7.30 & \\
\hline & p-coumaric acid & 0.49 & \\
\hline & gallic acid & 15.27 & \\
\hline & p-hydroxybenzoic acid & 0.80 & \\
\hline
\end{tabular}

Figure legend:

Fig. 1 . Therapeutic potential of coconut oil and its products in fighting COVID-19 caused by SARS-CoV-2 . The scheme depicts the utility of various coconut oil products in the development of an aerosol spray for use against SARS-CoV-2. The possible mode of action of lauric acid in disrupting the lipid membrane envelope of the virus and in blocking the interaction between viral Spike protein and human angiotensin converting enzyme (hACE) receptor are also presented.

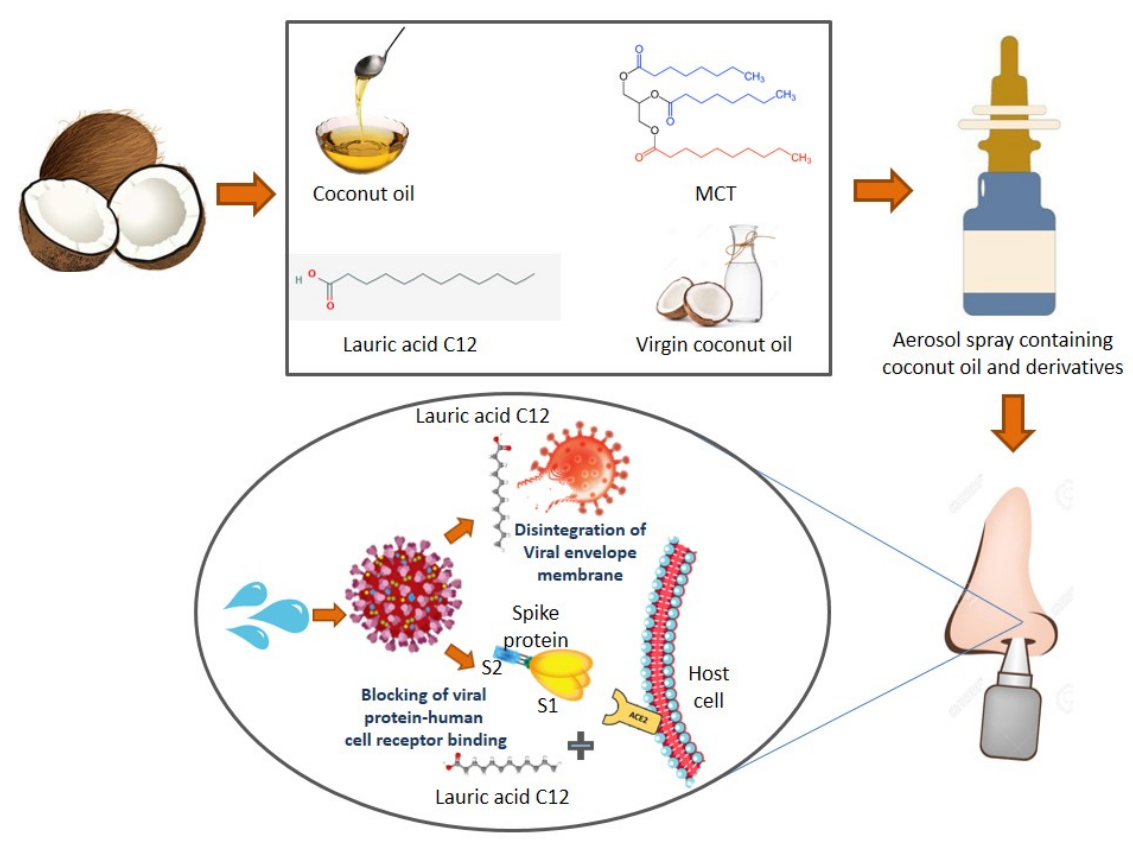

